\title{
Local Analytic Solutions of an Iterative Functional Differential Equation with Deviating Arguments Depending on the State Derivative
}

Jing Liu

Department of Mathematics, Binzhou University, Shandong, P. R. China

\begin{abstract}
In this paper, the existence of analytic solutions of a class of iterative functional differential equation with the form of $x^{\prime}(z)=x\left(a z+\frac{b}{x(z)}\right), z \in C$ is studied. By constructing a convergent power series solution of an auxiliary equation with the form of

$\left[a g^{\prime}(z)-\alpha g^{\prime}(\alpha z)\right]\left[g\left(\alpha^{2} z\right)-a g(\alpha z)\right]=[g(\alpha z)-a g(z)]^{2} g^{\prime}(z), z \in C$

the local analytic solutions for the original equation are obtained. The constant $\alpha$ obtained in the results is not only at resonance, i.e. at a root of the unity, but also near resonance (near a root of the unity) under Brjuno condition.
\end{abstract}

Keywords: Iterative functional differential equation; analytic solution; Diophantine condition; Brjuno condition.

2000 Mathematics Subject Classification: 39B12, 34A25, 30D05

\section{INTRODUCTION}

The functional differential equations with the form of

$x^{\prime}(z)=f(z, x(z-\sigma(z)))$,

have been lucubrated in $[1,2]$. However, such equations have been paid little attention to, which the delay function $\sigma(z)$ depends not only on the argument of the unknown function, but also the state $\sigma(z)=\sigma(z, x(z))$. In $[3,4]$, the authors studied the existence of analytic solution of the equations

$$
x^{\prime}(z)=x(a z+b x(z))
$$

and

$$
x^{\prime}(z)=x\left(a z+b x^{\prime}(z)\right)
$$

respectively. Taking $f(z, x)=x$ and $\sigma(z)=(1-a) z-\frac{b}{x(z)}$ in (1.1). we deduce the equation of the form

$x^{\prime}(z)=x\left(a z+\frac{b}{x(z)}\right), z \in C$

where $a$ and $b$ are complex. numbers $x(z)$ denotes the unknown complex function.

This paper discusses the existence of analytic solutions to (1.2) in the complex field. 
Jing Liu "Local Analytic Solutions of An Iterative Functional Differential Equation with Deviating Arguments Depending on the State Derivative"

Since such equations are quite different from the usual differential equations, the standard existence and uniqueness theorems cannot be applied directly. It is interested to find some and all of their solutions under appropriate conditions.

Set $a \neq 0, b=0$, Eq.(1.2) can be changed into functional differential equation

$$
x^{\prime}(z)=x(a z),
$$

which has an entire solution of the form(see Elbert[5])

$$
x(z)=\sum_{n=0}^{\infty} \frac{a^{\frac{n(n-1)}{2}}}{n !} \eta z^{n} .
$$

A distinctive feature of Eq.(1.2) is that the argument of the unknown function is dependent on the state $x(z)$ when $b \neq 0$.and this is the case we will emphasize in this paper. Let

$$
y(z)=a z+\frac{b}{x(z)},
$$

then

$$
x(z)=\frac{b}{y(z)-a z} .
$$

Therefore, in view of Eq.(1.3), $x^{\prime}(z)=\frac{b\left(a-y^{\prime}(z)\right)}{[y(z)-a z]^{2}}$ and $x(y(z))=\frac{b}{y(y(z))-a y(z)}$, we have

$$
\frac{b\left(a-y^{\prime}(z)\right)}{[y(z)-a z]^{2}}=\frac{b}{y(y(z))-a y(z)} .
$$

that is

$$
\left(a-y^{\prime}(z)\right)[y(y(z))-a y(z)]=[y(z)-a z]^{2} .
$$

In order to find the analytic solution of (1.4), as in previous works [6-13], our strategy remains to reduce Eq.(1.4) with $y(z)=g\left(\alpha g^{-1}(z)\right)$, which is called the Schröder transformation sometimes. To the auxiliary equation

$$
\left[a g^{\prime}(z)-\alpha g^{\prime}(\alpha z)\right]\left[g\left(\alpha^{2} z\right)-a g(\alpha z)\right]=[g(\alpha z)-a g(z)]^{2} g^{\prime}(z) \text {. }
$$

a functional differential equation with proportional delay, and discuss analytic solution of Eq.(1.5) with the initial value condition

$$
g(0)=\mu,
$$

where $\mu$ is a complex number.

Assume that $\alpha$ in Eq.(1.5) satisfies one of the following conditions:

$$
\left(H_{1}\right) 0<|\alpha|<1
$$

$\left(H_{2}\right) \alpha=e^{2 \pi i \theta}$, where $\theta \in R \backslash Q$ is a Brjuno number [14,15], i.e.

$B(\theta)=\sum_{k=0}^{\infty} \frac{\log q_{k+1}}{q_{k}}<+\infty$ where $\left\{p_{k} / q_{k}\right\}$ denotes the sequence of partial fraction of the continued fraction expansion of $\theta$, said to satisfy the Brjuno condition; 
Jing Liu "Local Analytic Solutions of An Iterative Functional Differential Equation with Deviating Arguments Depending on the State Derivative"

$\left(H_{3}\right) \alpha=e^{2 \pi i q / p}$, for some integer $p \in N$, with $p \geq 2$ and $q \in Z \backslash\{0\}$, and $\alpha \neq e^{2 \pi i l / k}$, for all $1 \leq k \leq p-1$, and $l \in Z \backslash\{0\}$.

Observe that $\alpha$ is inside the unit circle $S^{1}$ in the case of $\left(H_{1}\right)$ but on $S^{1}$ in the rest cases. More difficulties are encountered for $\alpha$ on $S^{1}$ since the small divisor $\alpha^{n}-1$ is involved in the later (2.9). Under Diophantine condition: " $\alpha=e^{2 \pi i \theta}$, where $\theta \in R \backslash Q$ and there exist constants $\zeta>0$ and $\sigma>0$ such that $\left|\alpha^{n}-1\right| \geq \zeta^{-1} n^{-\sigma}$ for all $n \geq 1$ ", The number $\alpha \in S^{1}$ is "far" from all roots of the unity, and was considered in different settings [11]. Since then, we have been striving to give a result in terms of analytic solutions for those $\alpha$ "near" a root of the unity, i.e., neither being roots of unity nor satisfying the Diophantine condition. The Brjuno condition in $\left(\mathrm{H}_{2}\right)$ provides such a chance for us, Moreover, we also discuss the so-called the resonance case. i.e.. the case of $\left(\mathrm{H}_{3}\right)$.

\section{ANALYTIC SOLUTION OF THE AUXILIARY EQUATION}

Theorem 2.1. Suppose $\left(H_{1}\right)$ holds and $a \neq 0,1, \alpha, b \neq 0$. Then for any $\eta \in C \backslash\{0\}$, Eq.(1.5) has an analytic solution with the form

$$
g(z)=\mu+\eta z+\sum_{n=2}^{\infty} b_{n} z^{n},
$$

where $\mu=\frac{a-\alpha}{1-a}$.

Proof We seek a solution of Eq.(1.5) in a power series of the form

$$
g(z)=\sum_{n=0}^{\infty} b_{n} z^{n},
$$

where $b_{0}=\mu$. By substituting (2.2) into (1.5), we get

$$
\begin{aligned}
& \sum_{n=0}^{\infty}\left(\sum_{i=0}^{n}(i+1)\left(a-\alpha^{i+1}\right) \alpha^{n-i}\left(\alpha^{n-i}-a\right) b_{i+1} b_{n-i}\right) z^{n} \\
& =\sum_{n=0}^{\infty}\left(\sum_{i=0}^{n} \sum_{k=0}^{n-i}(i+1)\left(\alpha^{k}-a\right)\left(\alpha^{n-i-k}-a\right) b_{i+1} b_{k} b_{n-i-k}\right) z^{n} .
\end{aligned}
$$

Comparing coefficients, we obtain

$(1-a)\left[(1-a) b_{0}-(a-\alpha)\right] b_{0} b_{1}=0$,

and

$$
\begin{aligned}
& \alpha(n+1)(a-\alpha)\left(1-\alpha^{n}\right) b_{n+1} \\
& =\sum_{i=0}^{n-1}(i+1)\left(\alpha^{i+1}-a\right) \alpha^{n-i}\left(\alpha^{n-i}-a\right) b_{i+1} b_{n-i}+\sum_{i=0}^{n-1} \sum_{k=0}^{n-i}(i+1)\left(\alpha^{k}-a\right)\left(\alpha^{n-i-k}-a\right) b_{i+1} b_{k} b_{n-i-k}, \\
& n=1,2, \cdots,
\end{aligned}
$$

In view of the definition of $\mu$, we see that $(1-a) b_{0}-(a-\alpha)=0$. So, we can choose $b_{1}$ to be $\eta$ in (2.3). Once $b_{0}$ and $b_{1}$ are determined, the other terms of the sequence $\left\{b_{n}\right\}_{n=0}^{\infty}$ can be determined successively from (2.4) in a unique manner. Now, we show that the power series (2.1) converges in a neighborhood of the origin. 
Jing Liu "Local Analytic Solutions of An Iterative Functional Differential Equation with Deviating Arguments Depending on the State Derivative"

Since $0<|\alpha|<1$, we have

$$
\left|\frac{(i+1)\left(\alpha^{k}-a\right)\left(\alpha^{n-i-k}-a\right)}{\alpha(n+1)(a-\alpha)\left(1-\alpha^{n}\right)}\right| \leq \frac{(1+|a|)^{2}}{|\alpha \| a-\alpha| 1-\alpha^{n} \mid} \leq M
$$

for some positive $M$. Thus, from (2.4) we obtain

$$
\left|b_{n+1}\right| \leq M\left(\sum_{i=0}^{n-1}\left|b_{i+1}\right|\left|b_{n-i}\right|+\sum_{i=0}^{n-1} \sum_{k=0}^{n-i}\left|b_{i+1} \| b_{k}\right|\left|b_{n-i-k}\right|\right), \quad n=1,2, \cdots,
$$

If we define a sequence $\left\{B_{n}\right\}_{n=0}^{\infty}$, by $B_{0}=|\mu|, B_{1}=|\eta|$, and

$$
B_{n+1}=M\left(\sum_{i=0}^{n-1} B_{i+1} B_{n-i}+\sum_{i=0}^{n-1} \sum_{k=0}^{n-i} B_{i+1} B_{k} B_{n-i-k}\right), \quad n=1,2, \cdots,
$$

then in view of (2.5), by induction, we can prove $\left|b_{n}\right| \leq B_{n}, n=1,2, \cdots$. Now, we define the function

$$
H(z, \mu, \eta, M)=\sum_{n=0}^{\infty} B_{n} z^{n},
$$

then

$$
\begin{aligned}
& H^{2}(z, \mu, \eta, M)=\sum_{n=0}^{\infty}\left(\sum_{k=0}^{n} B_{k} B_{n-k}\right) z^{n} \\
& =\left(|\mu|+\sum_{n=0}^{\infty} B_{n+1} z^{n+1}\right) \sum_{n=0}^{\infty} B_{n} z^{n} \\
& =|\mu| H(z, \mu, \eta, M)+\sum_{n=0}^{\infty}\left(\sum_{i=0}^{n} B_{i+1} B_{n-i}\right) z^{n+1} \\
& =|\mu| H(z, \mu, \eta, M)+|\mu||\eta| z+\sum_{n=1}^{\infty}\left(|\mu| B_{n+1}+\sum_{i=0}^{n-1} B_{i+1} B_{n-i}\right) z^{n+1} \\
& =2|\mu| H(z, \mu, \eta, M)-|\mu|^{2}+\sum_{n=1}^{\infty}\left(\sum_{i=0}^{n-1} B_{i+1} B_{n-i}\right) z^{n+1}
\end{aligned}
$$

$$
\begin{aligned}
& H^{3}(z, \mu, \eta, M) \\
& =\left(|\mu|+\sum_{n=0}^{\infty} B_{n+1} z^{n+1}\right)\left[\sum_{n=0}^{\infty}\left(\sum_{k=0}^{n} B_{k} B_{n-k}\right) z^{n}\right] \\
& =|\mu| H^{2}(z, \mu, \eta, M)+\sum_{n=0}^{\infty}\left(\sum_{i=0}^{n} \sum_{k=0}^{n-i} B_{i+1} B_{k} B_{n-i-k}\right) z^{n+1} \\
& =|\mu| H^{2}(z, \mu, \eta, M)+|\mu|^{2}|\eta| z+\sum_{n=1}^{\infty}\left(|\mu|^{2} B_{n+1} z^{n+1}+\sum_{n=1}^{\infty}\left(\sum_{i=0}^{n-1} \sum_{k=0}^{n-i} B_{i+1} B_{k} B_{n-i-k}\right) z^{n+1}\right) B_{n+1} z^{n+1} \\
& =|\mu| H^{2}(z, \mu, \eta, M)+|\mu|^{2}|\eta| z+|\mu|^{2}(H(z, \mu, \eta, M)-|\mu|-|\eta| z)+\sum_{n=1}^{\infty}\left(\frac{1}{M} B_{n+1}-\sum_{I=0}^{n-1} B_{i+1} B_{n-i}\right) z^{n+1} \\
& =(|\mu|-1) H^{2}(z, \mu, \eta, M)+\left(|\mu|^{2}+\frac{1}{M}-2|\mu|\right) H(z, \mu, \eta, M)-\frac{|\eta|}{M} z-\left(|\mu|^{2}+|\mu|^{3}+\frac{|\mu|}{M}\right),
\end{aligned}
$$

that is, 
Jing Liu "Local Analytic Solutions of An Iterative Functional Differential Equation with Deviating Arguments Depending on the State Derivative"

$H^{3}(z, \mu, \eta, M)-(|\mu|-1) H^{2}(z, \mu, \eta, M)-\left(|\mu|^{2}+\frac{1}{M}-2|\mu|\right) H(z, \mu, \eta, M)+\frac{|\eta|}{M} z+\left(|\mu|^{2}+|\mu|^{3}+\frac{|\mu|}{M}\right)=0$,

Define the function

$R(z, \varsigma, \mu, \eta, M)=\varsigma^{3}-(|\mu|-1) \varsigma^{2}-\left(|\mu|^{2}+\frac{1}{M}-2|\mu|\right) \varsigma+\frac{|\eta|}{M} z+\left(|\mu|^{2}+|\mu|^{3}+\frac{|\mu|}{M}\right)$.

For $(z, \varsigma)$ from a neighborhood of $(0,|\mu|)$. Since $R(0,|\mu|, \mu, \eta, M)=0$, and

$R_{\varsigma}^{\prime}(0,|\mu|, \mu, \eta, M)=\frac{1}{M}$, according to the implicit function theorem, there exists a unique function $\varsigma(z, \mu, \eta, M)$, analytic in a neighborhood of zero, such that

$\varsigma(0, \mu, \eta, M)=|\mu|, \quad \varsigma_{z}^{\prime}(0, \mu, \eta, M)=|\eta|$

and $R(z, \varsigma, \mu, \eta, M)=0$. By (2.6) and (2.7), we have $H(z, \mu, \eta, M)=\varsigma(z, \mu, \eta, M)$. It follows that the power series (2.6), and hence also (2.1), converges in a neighborhood of the origin. The proof is complete.

Next we devote to the existence of analytic solution of (1.6) under the Brjuno condition. To do this, we first recall briefly the definition of Brjuno numbers and some basic facts. As stated in [15], for a real number $\theta$, we let $[\theta]$ denote its integer part and $\{\theta\}=\theta-[\theta]$ denote its fractional part. Then every irrational number $[\theta]$ has a unique expression of the Gauss ', continued fraction

$\theta=a_{0}+\theta_{0}=a_{0}+\frac{1}{a_{1}+\theta_{1}}=\cdots$,

denoted simply by $\theta=\left[a_{0}, a_{1}, \cdots, a_{n}, \cdots\right]$, where $a_{j}^{\prime} \mathrm{s}$ and $\theta_{j}^{\prime} \mathrm{s}$ are calculated by the algorithm: (a) $a_{0}=[\theta], \theta_{0}=\{\theta\}$, and $(b) a_{n}=\left[\frac{1}{\theta_{n-1}}\right], \theta_{n}=\left\{\frac{1}{\theta_{n-1}}\right\}$ for all $n \geq 1$. Define the sequences $\left(p_{n}\right)_{n \in N}$ and $\left(q_{n}\right)_{n \in N}$ as follows:

$q_{-2}=1, \quad q_{-1}=0, \quad q_{n}=a_{n} q_{n-1}+q_{n-2}$,

$p_{-2}=0, \quad p_{-1}=1, \quad p_{n}=a_{n} p_{n-1}+p_{n-2}$.

It is easy to show that $p_{n} / q_{n}=\left[a_{0}, a_{1}, \cdots, a_{n}, \cdots\right]$. Thus, for every $\theta \in R \backslash Q$, we associate, using its convergence, an arithmetical function $B(\theta)=\sum_{n=0}^{\infty} \frac{\log q_{n+1}}{q_{n}}$. We say that $\theta$ is a Brjuno number or that it satisfies Brjuno condition if $B(\theta)<+\infty$. The Brjuno condition is weaker than the Diophantine condition. For example, if $a_{n+1} \leq c e^{a_{n}}$ for all $n \geq 0$, where $c>0$ is a constant, then $\theta=\left[a_{0}, a_{1}, \cdots, a_{n}, \cdots\right]$. is a Brjuno number but is not a Diophantine condition. So, the case $\left(H_{2}\right)$ contains both Diophantine condition and a part of $\alpha$ "near" resonance. Let $\theta \in R \backslash Q$ and $\left(q_{n}\right)_{n \in N}$ be the sequence of partial denominators of

the Gauss ', continued fraction for $\theta$. As in [15], let

$A_{k}=\left\{n \geq 0 \mid\|n \theta\| \leq \frac{1}{8 q_{k}}\right\}, \quad E_{k}=\max \left(q_{k}, \frac{q_{k+1}}{4}\right), \quad \eta_{k}=\frac{q_{k}}{E_{k}}$. 
Jing Liu "Local Analytic Solutions of An Iterative Functional Differential Equation with Deviating Arguments Depending on the State Derivative"

Let $A_{k}^{*}$ be the set of integers $j \geq 0$ such that either $j \in A_{k}$ or for some $j_{1}$ and $j_{2}$ in $A_{k}$, with $j_{1}-j_{2}<E_{k}$, One has $j_{1}<j<j_{2}$ and $q_{k}$ divides $j-j_{1}$. For any integer $n \geq 0$, define

$l_{k}(n)=\max \left(\left(1+\eta_{k}\right) \frac{n}{q_{k}}-2,\left(m_{n} \eta_{k}+n\right) \frac{1}{q_{k}}-1\right)$,

where $m_{n}=\max \left\{j \mid 0 \leq j \leq n, j \in A_{k}^{*}\right\}$. We then define function $h_{k}: N \rightarrow R_{+}$as follows:

$$
h_{k}(n)=\left\{\begin{array}{lll}
\frac{m_{n}+\eta_{k} n}{q_{k}}-1, & \text { if } & m_{n}+q_{k} \in A_{k}^{*}, \\
l_{k}(n), & \text { if } & m_{n}+q_{k} \notin A_{k}^{*} .
\end{array}\right.
$$

Let $g_{k}(n):=\max \left(h_{k}(n),\left[\frac{n}{q_{k}}\right]\right)$, and define $k(n)$ by the condition $q_{k(n)} \leq n \leq q_{k(n)+1}$. Clearly, $k(n)$ is non-decreasing. Then we are able to state the following result.

Lemma 2.1 (Davie 's lemma [16]) Let $K(n)=n \log 2+\sum_{k=0}^{k(n)} g_{k}(n) \log \left(2 q_{k+1}\right)$. Then

(a) there is a universal constant $\gamma>0$ (independent of $n$ and $\theta$ ), such that

$$
K(n) \leq n\left(\sum_{k=0}^{k(n)} \frac{\log q_{k+1}}{q_{k}}+\gamma\right)
$$

(b) $K\left(n_{1}\right)+K\left(n_{2}\right) \leq K\left(n_{1}+n_{2}\right)$ for all $n_{1}$ and $n_{2}$, and

$(c)-\log \left|\alpha^{n}-1\right| \leq K(n)-K(n-1)$.

Theorem2.2 Suppose $\left(H_{2}\right)$ holds and $a \neq 0,1, \alpha, b \neq 0$. Then for any $\eta \in C \backslash\{0\}$, Eq.(1.5) has an analytic solution of the form (2.1) in a neighborhood of the origin, where $\mu$ is the same number defined in theorem 2.1 .

Proof As in the proof of Theorem2.1, we seek a power series solution of the form (2.1). Set $b_{0}=\mu$ and $b_{1}=\eta$. Then (2.4) holds. From (2.4), we have

$\left|b_{n+1}\right| \leq \frac{L}{\left|1-\alpha^{n}\right|}\left(\sum_{i=0}^{n-1}\left|b_{i+1}\right|\left|b_{n-i}\right|+\sum_{i=0}^{n-1} \sum_{k=0}^{n-i}\left|b_{i+1}\left\|b_{k}\right\| b_{n-i-k}\right|\right), \quad n=1,2, \cdots$

where $L=\frac{(1+|a|)^{2}}{|\alpha||a-\alpha|}$. To construct a majorant series of (2.1), we consider the implicit functional equation

$R(z, \varphi, \mu, \eta, L)=0$,

where $R$ is defined in (2.8). Similarly to the proof of Theorem2.1, using the implicit function theorem we can prove that (2.10) has a unique analytic solution $\varphi(z, \mu, \eta, L)$ in a neighborhood of the origin such that $\varphi(0, \mu, \eta, L)=|\mu|$, and $\varphi_{z}^{\prime}(0, \mu, \eta, L)=|\eta|$, Thus

$\varphi(z, \mu, \eta, L)$ in (2.10) can be expanded into a convergent series

$\varphi(z, \mu, \eta, L)=\sum_{n=0}^{\infty} C_{n} z^{n}$ 
Jing Liu "Local Analytic Solutions of An Iterative Functional Differential Equation with Deviating Arguments Depending on the State Derivative"

in a neighborhood of the origin. Replacing (2.11) into (2.10) and comparing coefficients we obtain that $C_{0}=|\mu|, C_{1}=|\eta|$ and

$C_{n+1}=L\left(\sum_{i=0}^{n-1} C_{i+1} C_{n-i}+\sum_{i=0}^{n-1} \sum_{k=0}^{n-i} C_{i+1} C_{k} C_{n-i-k}\right), \quad n=1,2, \cdots$,

Note that the series (2.11) converges in a neighborhood of the origin. Hence there is a constant $T>0$ such that

$C_{n} \leq T^{n}, n=1,2, \cdots$.

Now, by induction, we can deduce that $\left|b_{n}\right| \leq C_{n} e^{K(n-1)}$ for $n \geq 1$, where $K: N \rightarrow R$ is

defined in Lamma 2.1. In fact $\left|b_{1}\right|=|\eta|=C_{1}$. For inductive proof, we assume that $\left|b_{j}\right| \leq C_{j} e^{K(j-1)}, j \leq m$. From (2.9) and Lamma 2.1

$$
\begin{gathered}
\left|b_{m+1}\right| \leq \frac{L}{\left|1-\alpha^{m}\right|}\left(\sum_{i=0}^{m-1}\left|b_{i+1}\right|\left|b_{m-i}\right|+\sum_{i=0}^{m-1} \sum_{k=0}^{m-i}\left|b_{i+1}\right|\left|b_{k}\right|\left|b_{m-i-k}\right|\right) \\
\leq \frac{L}{\left|1-\alpha^{m}\right|}\left(\sum_{i=0}^{m-1} C_{i+1} C_{m-i} e^{K(i)+K(m-i-1)}+\sum_{i=0}^{m-1} \sum_{k=0}^{m-i} C_{i+1} C_{k} C_{n-i-k} e^{K(i)+K(k-1)+K(n-i-k-1)}\right) .
\end{gathered}
$$

Note that

$$
K(i)+K(m-i-1) \leq K(m-1) \leq \log \left|\alpha^{m}-1\right|+K(m) .
$$

and

$$
K(i)+K(k-1)+K(m-i-k-1) \leq K(m-2) \leq K(m-1) \leq \log \left|\alpha^{m}-1\right|+K(m) .
$$

Then

$$
\left|b_{m+1}\right| \leq e^{K(m)}\left(\sum_{i=0}^{m-1} C_{i+1} C_{n-i}+\sum_{i=0}^{m-1} \sum_{k=0}^{m-i} C_{i+1} C_{k} C_{n-i-k}\right)=C_{m+1} e^{K(m)} .
$$

as desired. Note that $K(n) \leq n(B(\theta)+\gamma)$ for some universal constant $\gamma>0$. Then $\left|b_{n}\right| \leq T^{n} e^{(n-1)(B(\theta)+\gamma)}$,

that is

$$
\lim _{n \rightarrow \infty} \sup \left(\left|b_{n}\right|\right)^{\frac{1}{n}} \leq \lim _{n \rightarrow \infty} \sup \left(T^{n} e^{(n-1)(B(\theta)+\gamma)}\right)^{\frac{1}{n}}=T e^{B(\theta)+\gamma} .
$$

This implies that the convergence radius of $(2.1)$ is at least $\left(T e^{B(\theta)+\gamma}\right)^{-1}$. This completes the proof.

In case $\left(\mathrm{H}_{3}\right)$ the constant $\alpha$ is not only on the unit circle in $C$, but also a root of unity. In such a case, the resonant case, both Diophantine condition and Brjuno condition are not satisfied.

Let $\left\{D_{n}\right\}_{n=0}^{\infty}$ be a sequence define by $D_{0}=|\mu|, D_{1}=|\eta|$ and

$$
D_{n+1}=\Gamma L\left(\sum_{i=0}^{n-1} D_{i+1} D_{n-i}+\sum_{i=0}^{n-1} \sum_{k=0}^{n-i} D_{i+1} D_{k} D_{n-i-k}\right), \quad n=1,2, \cdots
$$


Jing Liu "Local Analytic Solutions of An Iterative Functional Differential Equation with Deviating Arguments Depending on the State Derivative"

Where $\Gamma=\max \left\{1,\left|\alpha^{i}-1\right|^{-1}: i=1,2, \cdots, p-1\right\} \quad$ and $L$ is defined in Theorem 2.2.

Theorem2.3 Suppose $\left(H_{3}\right)$ holds and $a \neq 0,1, \alpha, b \neq 0$, and $p$ is given as above. Let $\left\{b_{n}\right\}_{n=0}^{\infty}$ be determined recursively by $b_{0}=\mu, b_{1}=\eta$ and

$\alpha(n+1)(a-\alpha)\left(1-\alpha^{n}\right) b_{n+1}=\Phi(n, \alpha), \quad n=1,2, \cdots$.

where

$$
\Phi(n, \alpha)=\sum_{i=0}^{n-1}(i+1)\left(\alpha^{i+1}-a\right) \alpha^{n-i}\left(\alpha^{n-i}-a\right) b_{i+1} b_{n-i}+\sum_{i=0}^{n-1} \sum_{k=0}^{n-i}(i+1)\left(\alpha^{k}-a\right)\left(\alpha^{n-i-k}-a\right) b_{i+1} b_{k} b_{n-i-k}
$$

If $\Phi(v p, \alpha)=0$ for all $v=1,2, \cdots$, then Eq.(1.5) has an analytic solution of the form

$$
g(z)=\mu+\eta z+\sum_{n=v p+1, v \in N} \xi_{v p+1} z^{n}+\sum_{n \neq v p+1, v \in N} b_{n} z^{n}, \quad N=\{1,2,3, \cdots\}
$$

in a neighborhood of the origin, where all $\xi_{v p+1}^{\prime} s$ are arbitrary constants satisfying the inequality $\left|\xi_{v p+1}\right| \leq D_{v p+1}$ and the sequence $\left\{D_{n}\right\}_{n=0}^{\infty}$ is defined in (2.14). Otherwise, if $\Phi(v p, \alpha) \neq 0$ for some $v=1,2, \cdots$, then Eq.(1.5) has no analytic solutions in any neighborhood of the origin.

Proof We seek a power series solution of (1.5) of the form (2.1) as in the proof of Theorem 2.1, where the equality (2.4) or (2.5) is satisfied. If $\Phi(v p, \alpha) \neq 0$ for some natura number $v$, then the equality in (2.15) does not hold for $n=v p$ since $1-\alpha^{v p}=0$. In such a circumstance Eq.(1.5) has no formal solutions.

When $\Phi(v p, \alpha)=0$ for all natural number $v$, for each $v$ the corresponding $b_{v p+1}$ in (2.15) has infinitely many choices in $C$, that is, the formal series solution (2.1) defines a family of solutions with infinitely many parameters. Choose $b_{v p+1}=\xi_{v p+1}$ arbitrarily such that

$\left|\xi_{v p+1}\right| \leq D_{v p+1}, \quad v=1,2, \cdots$

Where $D_{v p+1}$ is defined by (2.14). In what follows we prove that the formal series solution (2.1) converges in a neighborhood of the origin. Observe that $\left|\alpha^{n}-1\right|^{-1} \leq \Gamma$ for $n \neq v p$. It follows from (2.15) that

$\left|b_{n+1}\right| \leq \Gamma L\left(\sum_{i=0}^{n-1}\left|b_{i+1}\right|\left|b_{n-i}\right|+\sum_{i=0}^{n-1} \sum_{k=0}^{n-i}\left|b_{i+1}\right|\left|b_{k}\right|\left|b_{n-i-k}\right|\right)$

for all $n \neq v p, v=1,2, \cdots$.

Let

$W(z, \mu, \eta, \Gamma, L)=\sum_{n=0}^{\infty} D_{n} z^{n}, \quad D_{0}=|\mu|, \quad D_{1}=|\eta|$.

It is easy to check that (2.18) satisfies the implicit functional equation

$R(z, \psi, \mu, \eta, \Gamma, L)=0$, 
Jing Liu "Local Analytic Solutions of An Iterative Functional Differential Equation with Deviating Arguments Depending on the State Derivative"

Where $R$ is defined in (2.8). Moreover, similarly to the proof of Theorem 2.1, we can prove that (2.19) has a unique analytic solution $\psi(z, \mu, \eta, \Gamma, L)$ in a neighborhood of the origin such that $\psi(0, \mu, \eta, \Gamma, L)=|\mu|$ and $\psi_{z}^{\prime}(0, \mu, \eta, \Gamma, L)=|\eta|$. Moreover, we also have

$\psi(z, \mu, \eta, \Gamma, L)=W(z, \mu, \eta, \Gamma, L)$. Thus (2.18) converges in a neighborhood of the origin. Moreover, it is easy to show that, by induction,

$$
\left|b_{n}\right| \leq D_{n}, \quad n=1,2, \cdots .
$$

Therefore, the series (2.1) converges in a neighborhood of the origin. This completes the proof.

ANALYTIC SOLUTION OF (1.2)

Theorem3.1 Under one of the conditions in Theorem 2.1-2.3, Eq.(1.4) has an analytic solution of the form $y(z)=g\left(\alpha g^{-1}(z)\right)$ in a neighborhood of the number $\mu$, where $g(z)$ is an analytic solution of (1.5).

Proof By Theorem 2.1-2.3, we may find an analytic solution $g(z)$ of the auxiliary equation (1.5) in the form of (2.1) such that $g(0)=\mu$ and $g^{\prime}(0)=\eta \neq 0$. Clearly the inverse

$g^{-1}(z)$ exists and is analytic in a neighborhood of $g(0)=\mu$. If we define $y(z)$ by means of $g\left(\alpha g^{-1}(z)\right)$, then

$$
\begin{aligned}
& y^{\prime}(z)=\frac{\alpha g^{\prime}\left(\alpha g^{-1}(z)\right)}{g^{\prime}\left(g^{-1}(z)\right)}, \\
& \left(a-y^{\prime}(z)\right)[y(y(z))-a y(z)] \\
& =\frac{\left[a g^{\prime}\left(g^{-1}(z)\right)-\alpha g^{\prime}\left(\alpha g^{-1}(z)\right)\right]\left[g\left(\alpha^{2} g^{-1}(z)\right)-a g\left(\alpha g^{-1}(z)\right)\right]}{g^{\prime}\left(g^{-1}(z)\right)} \\
& =\frac{\left[g\left(\alpha g^{-1}(z)\right)-a g\left(g^{-1}(z)\right)\right]^{2} g^{\prime}\left(g^{-1}(z)\right)}{g^{\prime}\left(g^{-1}(z)\right)} \\
& =[y(z)-a z]^{2} .
\end{aligned}
$$

as required. This completes the proof.

It has been shown that under the conditions of Theorem 2.1, 2.2 or 2.3, Eq.(1.4) has an analytic solution $y(z)=g\left(\alpha g^{-1}(z)\right)$ in a neighborhood of the number $\mu$, where $g(z)$ is an analytic solution of (1.5). Since the function $g(z)$ in (2.1) can be determined by (2.4), it is possible to calculate, at least in theory, the explicit form of $y(z)$, an analytic solution of (1.2), in a neighborhood of the fixed point $\mu$ of $y(z)$ by means of (1.3). However, knowing that an analytic solution of (1.2) exists, we can take an alternative route as follows. Assume that $x(z)$ is of the form

$$
x(z)=x(\mu)+x^{\prime}(\mu)(z-\mu)+\frac{x^{\prime \prime}(\mu)}{2 !}(z-\mu)^{2}+\cdots ;
$$

We need to determine the derivatives $x^{(n)}(\mu), n=0,1,2, \cdots$. First of all, in view of (1.3), we have

$$
x(\mu)=\frac{b}{y(\mu)-a \mu}=\frac{b}{(1-a) \mu} .
$$

and

$$
x^{\prime}(\mu)=x\left(a \mu+\frac{b}{x(\mu)}\right)=x(a \mu+(1-a) \mu)=x(\mu)=\frac{b}{(1-a) \mu} .
$$


Jing Liu "Local Analytic Solutions of An Iterative Functional Differential Equation with Deviating Arguments Depending on the State Derivative"

Next by calculating the derivatives of both of (1.2), we obtain

$$
x^{\prime \prime}(z)=x^{\prime}\left(a z+\frac{b}{x(z)}\right)\left(a-\frac{b x^{\prime}(z)}{(x(z))^{2}}\right) .
$$

Thus

$$
x^{\prime \prime}(\mu)=x^{\prime}(\mu)[a-(1-a) \mu]=\frac{b[a-(1-a) \mu]}{(1-a) \mu} .
$$

It seems from above that the higher derivatives $x^{(m)}(z)$ at $z=\mu$ can be determined uniquely in similar manners. Hence let $x^{(m)}(z)=\lambda_{m}$, it is easy to write the explicit form of our solution $x(z)$

$$
x(z)=\frac{b}{(1-a) \mu}+\frac{b}{(1-a) \mu}(z-\mu)+\frac{b[a-(1-a) \mu]}{2 !(1-a) \mu}(z-\mu)^{2}+\sum_{n=3}^{\infty} \frac{\lambda_{n}}{n !}(z-\mu)^{n} .
$$

\section{REFERENCES}

[1] J. Hale, Theory of Functional Differential Equations, Springer-Verlag, 1977.

[2] R. Bellman, K.Cooke, Differential-Difference Equations, Academic Press, 1963.

[3] J. Si, Sui-Sun Cheng. Analytic solutions of a functional differential equation with state dependent argument [J]. Taiwanese journal of mathematics, 1997, 1(4):471-480.

[4] F.Qiu, X.K.Dou, Analytic solutions of a first order iterative differential equation [J]. Journal of binzhou university, 2005, 21(6):11-14.

[5] A.Elbert, Asymptotic behaviour of the analytic solution differential equation $y(q u)=0$ as $q \rightarrow 1^{-}[\mathrm{J}]$. Comput Appl. Math, 1992, 41:5-22.

[6] Z. Jackiewicz, Existence and uniqueness of solutions of neutral delay-differential equations with state dependent delays[J], Funkcial. Ekvac. 30 (1987): 9-17.

[7] F. Qiu, H.Z. Liu, Analytic solutions of a first order iterative differential equation [J]. Demonstratio Mathematica 1 (2006): 81-90.

[8] F. Qiu, M. Su, Analytic solutions of a first order iterative differential equation [J]. Journal of Liaocheng University. 18(4) (2005): 18-23.

[9] J. Si,W. Li, S. Cheng, Analytic solutions of an iterative functional differential equation[J]. Comput. Math. Appl. 33(6) (1997): 47-51

[10] J. Si, S. Cheng, Note on an iterative functional differential equation [J]. Demostratio Math. 31 (3) (1998): 599-614.

[11] X. Wang, J. Si, Analytic solutions of an iterative functional differential equation[J]. Math. Anal. Appl. 262 (2001): 490-498.

[12] J. Si, W. Zhang, Analytic solutions of a class of iterative functional differential equations [J]. Comput. Appl. Math. 162 (2004): 467-481.

[13] J. Si, X. Wang, S. Cheng, Analytic solutions of a functional differential equation with a state derivative dependent delay [ J]. Aequationes Math. 57 (1999): 75-86.

[14] A.D. Brjuno, Analytic form of differential equations [J]. Trans. Moscow Math. Soc. 25 (1971): 131-288.

[15] T. Carletti, S. Marmi, Linearization of analytic and non-analytic germs of diffeomorphisms of (C, 0)[J]. Bull. Soc. Math. France, 128 (2000) : 69-85.

[16] A.M. Davie, The critical function for the semistandard map [J]. Nonlinearity 7 (1994): 219-229. 
Jing Liu "Local Analytic Solutions of An Iterative Functional Differential Equation with Deviating Arguments Depending on the State Derivative"

\section{AUTHOR'S BIOGRAPHY}

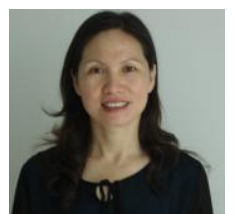

Jing Liu, Jing Liu is an associate professor at the Department of Mathematics in Binzhou University, Shandong, PRC. Her research interests include analytic solution and differential equation theory. 